\title{
BENEFICIOS DEL ROL DE ENFERMERÍA EN SALUD ESCOLAR, IMPLEMENTACIÓN A NIVEL INTERNACIONAL. REVISIÓN SISTEMÁTICA
}

\author{
BENEFITS OF THE NURSING ROLE IN SCHOOL HEALTH, IMPLEMENTATION AT \\ INTERNATIONAL LEVEL. SYSTEMATIC REVIEW
}

\section{María Francisca Cáceres Pérez}

Licenciada en Enfermería

Facultad de Medicina Universidad Católica del Norte, La Serena, Chile maria.caceres@alumnos.ucn.cl https://orcid.org/0000-0002-8126-3974

\section{Nicole Isabel Castillo Castillo}

Licenciada en Enfermería

Facultad de Medicina Universidad Católica del Norte, La Serena, Chile nicole.castillo01@alumnos.ucn.cl https://orcid.org/ORCID 0000-0002-8580-1899

\section{Camila Fernanda Salinas Vergara}

Licenciada en Enfermería

Facultad de Medicina Universidad Católica del Norte, La Serena, Chile csv015@alumnos.ucn.cl https://orcid.org/0000-0003-0592-1901

\section{Andrés Felipe Bianchetti Saavedra}

Antropólogo, Magister en Educación Mención Pedagogía y Gestión Universitaria Facultad de Medicina Universidad Católica del Norte, La Serena, Chile andres.bianchetti@ucn.cl https://orcid.org/0000-0002-6607-7380

\section{Rosa Inés Vega Flores}

Enfermera, Dra. en Enfermería

Facultad de Medicina Universidad Católica del Norte, La Serena, Chile rvega@ucn.cl

https://orcid.org/0000-0002-1576-1705

Artículo recibido el 30 de marzo de 2021. Aceptado en versión corregida el 30 de julio de 2021. 
Beneficios del rol de enfermería en salud escolar...

\title{
RESUMEN
}

La salud escolar en Chile se desarrolla a nivel de la Atención Primaria en salud a cargo de los profesionales de enfermería, quienes por su rol deben velar por el bienestar integral de la comunidad escolar, fortaleciendo conductas de autocuidado y hábitos saludables a través de la promoción y prevención e investigación en salud. OBJETIVO: Analizar los beneficios del rol de enfermería en salud escolar desde el punto de vista político y social en países en que ha sido legalmente implementada a nivel internacional. MÉTODOS: Se realizó una revisión sistemática de la literatura científica en bases de datos (Scielo, Pubmed, Scopus, Sciencedirect, Oxford, Web of Science). Se utilizó el flujograma PRISMA. La calidad metodológica consideró los criterios de alto, medio y bajo cumplimiento de 46 indicadores de una pauta de lectura crítica. RESULTADOS: Se identificaron 32 artículos en los últimos 5 años. Las líneas temáticas encontradas fueron las políticas públicas en salud escolar, destacando países como EEUU, España y México con regulación sobre la Enfermera escolar, en cambio en Latinoamérica, existe una regulación parcial o carencia como en Chile. Las competencias y acciones del profesional de enfermería en establecimientos educacionales tienen como prioridad la promoción de la salud y la educación a la comunidad escolar. Se evidencia que las consultas que atiende la enfermera escolar son de urgencia y son variables según la zona geográfica. CONCLUSIONES: La implementación legal del rol de enfermería escolar ofrece ventajas significativas para la mejora del sistema sanitario del país, incrementando la calidad asistencial y mejorando el acceso a salud.

Palabras clave: Servicios de Enfermería Escolar, Servicios de Salud Escolar, Educación en Salud, Rol de la Enfermera, Enfermería en salud comunitaria.

\begin{abstract}
Chilean school health is developed at the level of Primary Health Care in charge of nursing professionals, who for their role must ensure the comprehensive well-being of the school community, strengthening self-care behaviors and healthy habits through promotion and prevention and health research. OBJECTIVE: To analyze the benefits of the nursing role in school health from the political and social point of view in countries where it has been legally implemented internationally. METHODS: A systematic review of the scientific literature in databases (Scielo, Pubmed, Scopus, Sciencedirect, Oxford, Web of Science) was carried out. The PRISMA flow chart was used. The methodological quality considered the criteria of high, medium and low compliance with 46 indicators of a critical reading pattern. RESULTS: 32 articles were identified in the last 5 years. The thematic lines found were public policies in school health, highlighting countries such as the US, Spain and Mexico with regulations on school nurses, on the other hand in Latin America, there is a partial regulation or lack as in Chile. The competences and actions of the nursing professional in educational establishments have as a priority the promotion of health and education to the school community. It is evident that the consultations attended by the school nurse are urgent and vary according to the geographical area. CONCLUSIONS: The legal implementation of the
\end{abstract}


role of school nursing offers significant advantages for the improvement of the country's health system, increasing the quality of care and improving access to health.

Keywords: School Nursing, School Health Services, Health Education, Nurse's role, Community health nursing.

\section{http://dx.doi.org/10.7764/Horiz_Enferm.32.2.192-211}

\section{INTRODUCCIÓN}

La salud escolar existe desde principios del siglo XX en diversos países, tales como Estados Unidos, Australia y del entorno europeo: Francia, Suiza, Inglaterra. En 1944 se establece la necesidad de la educación para los establecimientos escolares por lo que surge en Chile la Ley de Salud Escolar que involucra la prevención, promoción y educación de la salud dentro del ámbito educativo. Actualmente nos encontramos en un aumento de patologías crónicas durante la infancia complicadas de tratar las cuales afectan en la formación, escolarización y su desarrollo psicoemocional. Estas necesidades justifican la implantación de programas educativos y la figura de un profesional de la salud competente $^{(1)}$. Sin embargo, en algunos países como España, aún no se consolida el rol de la enfermera en los establecimientos educacionales, quedando establecido sólo un número limitado de comunidades con el desempeño del rol de enfermería definido (por ejemplo, en Madrid y Andalucía) y no se encuentran referentes teóricos para la enfermería escolar (1). Por otro lado, la situación es claramente distinta en países latinoamericanos como Chile, Argentina, Bolivia y Brasil donde no existen profesionales de enfermería que cumplan con el rol en salud escolar en los establecimientos educacionales. En Latinoamérica existe evidencia que las enfermeras de atención primaria asumen algunas funciones del cuidado de enfermería escolar, insertas en el programa de salud escolar ${ }^{(2)}$.

La estrategia de municipios, comunas y comunidades saludables ha sido implementada en varios países de Europa (Irlanda, España, Finlandia entre otros) y en Latinoamérica: Perú, Argentina y México. Esta estrategia busca posicionar la promoción de la salud como la más alta prioridad de la agenda política y tiene el objetivo de "fortalecer el rol del municipio, como actor estratégico, para mejorar los resultados en salud y la calidad de vida de la población, en conjunto con la comunidad e intersectorialidad"(2).

En el caso de Chile el año 2013 se promulgó la Ley N²0,670 que creó el programa Elige Vivir Sano con el objetivo de promover hábitos y estilos de vida saludables. Hoy en día este programa tiene la finalidad de "contribuir al desarrollo de políticas públicas de nivel nacional, regional y comunal que construyan oportunidades para que las personas, familias $y$ comunidades accedan a entornos saludables"(3), dentro del cual se identifican tres entornos prioritarios para 
Beneficios del rol de enfermería en salud escolar...

el desarrollo de la promoción de la salud: comunal, educacional y laboral $^{(3)}$.

En el año 2015 surge el programa de Apoyo a Buenas Prácticas de Promoción de la Salud en el modelo de atención de salud integral familiar y comunitaria en la atención primaria y establecimientos educacionales, que potenció el modelo de atención integral de salud $^{(2)}$. Posteriormente se integran los establecimientos educacionales promotores de salud (EEPS) con el objetivo de la creación de entornos saludables dentro de los establecimientos, otorgando condiciones ambientales y garantizando la salud de su comunidad educativa, para acceder a un ambiente saludable y seguro. En este contexto, los establecimientos realizan actividades de promoción de la salud específicamente en áreas de alimentación saludable, actividad física, educación y salud sexual, prevención del consumo de alcohol, tabaco y otras drogas, salud bucal y lactancia materna. En este contexto, se construye una alianza entre los centros de salud y los establecimientos educacionales, vinculado con las orientaciones de establecimientos educacionales promotores de salud (EEPS) y el Plan de Educación Municipal $^{(3)}$.

En Chile, existe la Sociedad Chilena de Enfermería y Salud Escolar (SOCHIESE), corporación que reúne a enfermeras interesadas en contribuir al adecuado desarrollo de la gestión del cuidado del escolar en el nivel primario de atención de salud, así con el objetivo de mejorar el nivel de la salud escolar en el país ${ }^{(4)}$. Su función principal es la gestión del cuidado en la promoción, la mantención y restauración de la salud, y la prevención de enfermedades y lesiones ${ }^{(5)}$.
El Programa de Salud Escolar está direccionado a mantener estilos de vida saludable y promoción de la salud en los establecimientos educacionales, pero no aseguran la atención de salud a través de la enfermera escolar ${ }^{(6)}$. Si bien la enfermera de atención primaria de salud (APS) abarca estos elementos del programa, no está en forma permanente en los colegios y las actividades de promoción y prevención son limitadas. Esto es a excepción de países como Colombia y Perú donde los profesionales de enfermería tienen una función del rol definida y se encargan sobre todo de la prevención y promoción de la salud ${ }^{(7)}$.

Desde esta realidad presentada, que es heterogénea, surge la motivación y la idea de estudio para analizar los beneficios del rol de enfermería a nivel internacional. El entorno escolar es un lugar que permite ejercer las 6 áreas del quehacer de enfermería: asistencial, gestión, educación, emancipación, política e investigación y junto con ello la prevención y promoción de la salud de los escolares.

El propósito de esta revisión es el de analizar los beneficios del rol de enfermería en salud escolar desde el punto de vista político y social en los países en que ha sido legalmente implementada a nivel internacional orientadas a identificar los beneficios de la enfermería en los colegios.

\section{MÉTODOS}

Estudio de revisión sistemática cualitativa guiado por la declaración PRISMA (Preferred Reporting Items for Systematic Reviews and Meta-Analyses) para los métodos de búsqueda, selección, 
cribado e inclusión. Se realizó una búsqueda bibliográfica exploratoria en 6 bases de datos, las cuales contienen información almacenada de publicaciones internacionales de artículos científicosmédicos e información sobre la enfermería escolar. Los términos utilizados para la búsqueda estuvieron conformados por "servicios de enfermería escolar", "servicios de salud escolar", "educación en salud,", "rol de la enfermera" y "enfermería en salud comunitaria" [DeCS] se consideraron en la composición de descriptores y ecuaciones booleanas [OR, AND], que permitieron la selección de artículos publicados según criterios de inclusión y exclusión. Los criterios de inclusión fueron: (i) idioma: artículos en español, inglés y portugués, (ii) año de publicación: entre los años 2015-2020, (iii) accesibilidad: artículos exentos de pagos para su visualización y (iv) artículos de tipo cualitativo y cuantitativo, revisión sistemática y metaanálisis. Luego, los criterios de exclusión: (i) artículo publicado hace más de 6 años (ii) artículos con costo económico para su acceso, (iii), artículos en idioma que difiere a los del criterio de inclusión, (iv) artículos de revisión narrativa, ensayos y carta al editor y (v) artículos que no se relacionan con la enfermería en salud escolar se aplicaron en dos etapas (lectura de títulos y resumen del texto). Se excluyeron de la investigación las revisiones de literatura narrativa, ensayos y cartas al director.

El periodo de tiempo realizado fue desde el 4 de junio 2020 hasta el 18 enero 2021. Su distribución por base de datos son las siguientes:

En Scielo se encontraron 4.978 artículos, Pubmed se identificaron 515 artículos, en Scopus 231 artículos, Sciencedirect 5.891 artículos, Oxford 34.529 artículos y Web of Science 3.011 artículos. Por lo tanto, los artículos totales identificados en las 6 bases de datos fueron 49.228. Se identificaron un total de 50 citas duplicadas, que se excluyeron, quedando un total de 49.178 artículos.

Posteriormente se hizo el cribado de 2 formas: por título y por resumen. En la base de datos de Scielo se seleccionaron 36 artículos por título de los cuales fueron 16 elegidos por resumen. Se eliminaron 4.932 artículos. En la base de datos de Pubmed se seleccionaron 12 artículos por título de los cuales fueron elegidos 4 artículos por resumen. Se eliminaron 506 artículos. En la base de datos de Scopus se seleccionaron 27 artículos por título de los cuales fueron elegidos 19 artículos por resumen. Se eliminaron 208 artículos. En la base de datos de Sciencedirect se seleccionaron 65 artículos por títulos de los cuales se seleccionaron 18 artículos por resumen. Se eliminaron 5.863 artículos. En la base de datos de Oxford se seleccionaron 15 artículos por título de los cuales fueron elegidos 4 artículos por resumen. Se eliminaron 34.525 artículos. En la base de datos de Web of Science se seleccionaron 19 artículos por título de los cuales se seleccionaron 10 artículos por resumen. Se eliminaron 3.001 artículos.

Esto dio un total de 247 artículos por título de los cuales fueron seleccionados 96 artículos por resumen. Las autoras leyeron los 96 artículos a texto completo, descartando 63 artículos porque no tenían relación con los objetivos de la revisión sistemática, eligiendo 32 artículos que sí tenían relación con los objetivos establecidos. 
Tabla 1. Flujograma de búsqueda PRISMA y selección de estudios. Fuente: Elaboración propia.

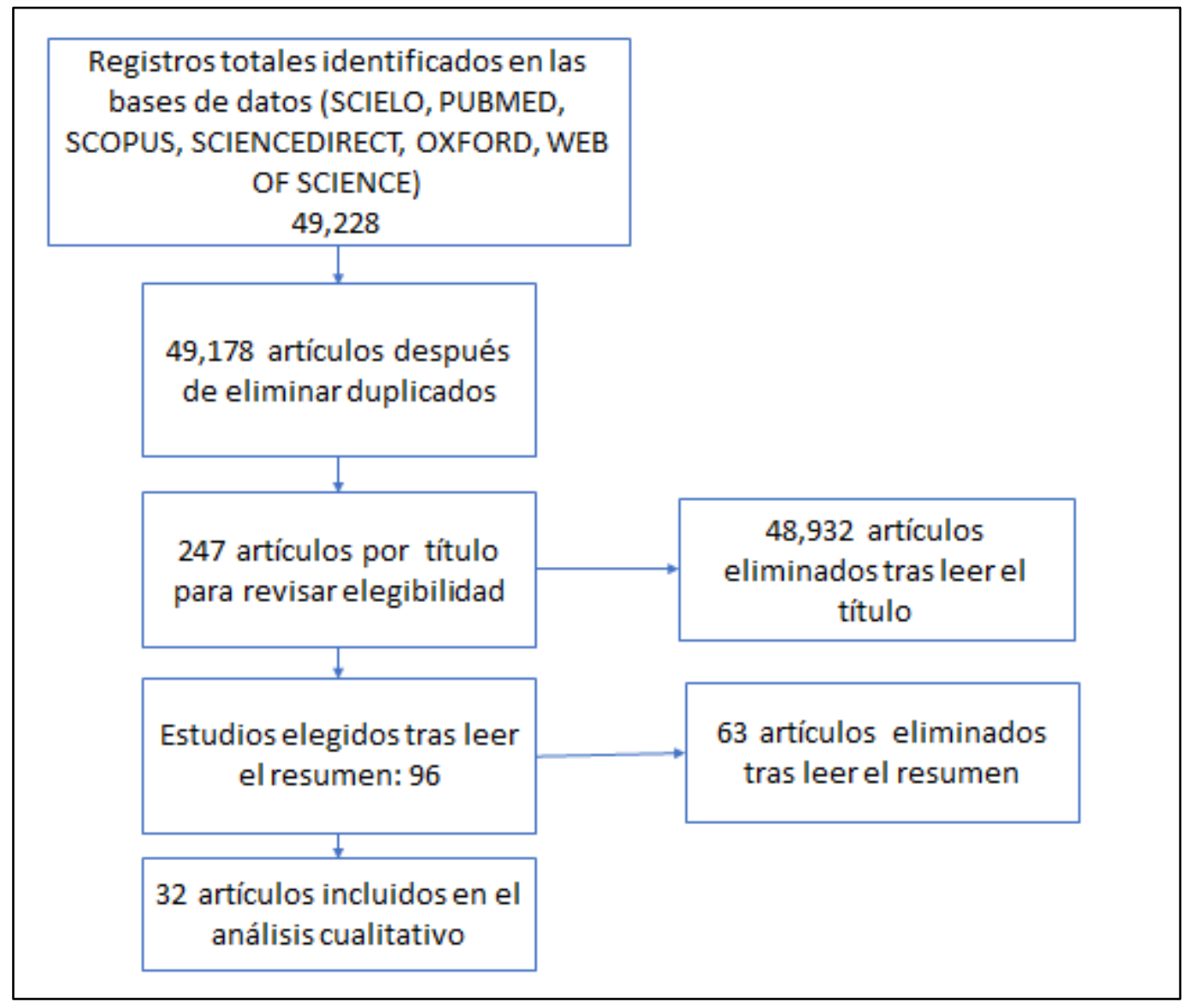

Para determinar la evaluación de la calidad metodológica, se utilizó la "Guía práctica de lectura crítica de artículos científicos originales en ciencias de la Salud"(8), de la cual se rescataron 3 indicadores por ítem sobre la estructura de un artículo. Se diseñó una pauta nueva de indicadores. Para la evaluación de cada artículo se consideraron 46 indicadores. Luego se trasladó a una matriz de datos, donde se diseñó el check list en donde se identifica si los indicadores establecidos cumplen con lo estipulado en la pauta de evaluación. Determinando que con $\geq 37$ indicadores el artículo se clasificó como de alta calidad (80\%). Con 23 a 36 indicadores: calidad media (78\%); y con 0 a 22 indicadores: calidad baja $(<50 \%)$.

Posteriormente se incluyeron solo los que cumplían con calidad metodológica alta y media. El número total de artículos incluidos en la revisión sistemática fueron 32. El número total de artículos excluidos en la síntesis de la revisión sistemática fue 1 .

Para evaluar el nivel de evidencia de los artículos se utilizó la escala de Sackett ${ }^{(9)}$, la cual clasifica los niveles de evidencia en 5 grados según letras de escala: $\mathrm{ABCDE}$, en donde la clasificación "A" corresponde al mejor nivel de evidencia y la clasificación "D" al nivel de evidencia más bajo. 
Finalmente, se utilizó una matriz de datos que permitió organizar la información y sintetizar la evidencia presentada para su posterior análisis.

\section{RESULTADOS Y DISCUSIÓN}

En la revisión sistemática se analizaron un total de 33 artículos, de los cuales se seleccionaron 32. La mayoría fue de calidad metodológica alta y media (97\%), a excepción de 1 artículo (3\%) el cual fue excluido de la revisión.

El idioma predominante de los estudios revisados fue el español con un total de 19 artículos, seguido del idioma inglés con 10 artículos revisados, luego el portugués con 3 artículos. La metodología cualitativa estuvo presente en 17 artículos, mientras que la cuantitativa en 7 , por otra parte, los artículos de metodología mixta fueron 8. En su mayoría los estudios fueron desarrollados por internas de enfermería, profesionales de enfermería y de otras áreas de la salud, también se presentan a menudo investigadores dedicados a la docencia. Los años de publicación: entre 2015 y 2020, incluye países a nivel internacional de los cuales se obtuvieron 9 en España, 5 en Chile, 3 en Brasil, 3 en EE.UU., 2 en Turquía, 2 en Inglaterra, 2 en Colombia, 1 en Polonia, 1 en Portugal, 1 en Finlandia, 1 en Canadá, 1 en México, 1 en Perú.

Luego de realizar un análisis de cada una de las investigaciones encontradas en la literatura, se evidencia que la temática se divide en 4 categorías que son las siguientes: políticas públicas en salud escolar, competencias y acciones del profesional de enfermería en los establecimientos educacionales, perspectivas de la comunidad escolar frente a la enfermería profesional en salud escolar y aspectos sociales del rol de profesionales de enfermería en salud escolar.

\section{Políticas públicas en salud escolar a nivel internacional}

Las políticas públicas incorporadas a nivel internacional presentan diferencias, ya que hay países que presentan una regulación nacional sobre la enfermería escolar tales como EE.UU., España, México y Colombia los cuales poseen políticas públicas en los establecimientos escolares sobre el rol de la enfermera escolar con sus correspondientes funcionalidades. Por otro lado, en países europeos y latinoamericanos se presenta una regulación parcial o una carencia desde el punto de vista de las funciones de enfermería en salud escolar. La literatura reporta, según Hernández et al. ${ }^{(10)}$, que en Latinoamérica en muy pocos casos se ha propiciado la incorporación de acciones por profesionales de la salud a los proyectos educativos o a los planes de estudios. Sumado a esto, en un estudio realizado por Martínez et al. ${ }^{(11)}$, en España, cuyo objetivo fue determinar el grado de consenso de expertos en salud pública sobre las funciones de la enfermería escolar en el ámbito asistencial y educación en salud, se aprecia que no existe un reconocimiento o regulación de los servicios de enfermería escolar en todas las comunidades autónomas por igual, además de una falta de regulación de la especialidad propia y que habitualmente el rol de la enfermera escolar no está presente en los colegios. La necesidad de regular los programas de salud escolar y la presencia de enfermeras escolares es evidenciada por García et al. ${ }^{(12)}$, en un 
Beneficios del rol de enfermería en salud escolar...

estudio realizado en España, que presentó la necesidad de enfermeras en establecimientos educacionales ya que a través de una encuesta a adolescentes arrojaron que las principales problemáticas de salud son el sobrepeso y la obesidad, por lo tanto, es importante tener una figura que posea el rol de promover la salud en el ámbito escolar. Esto es debido a que la adolescencia es una etapa clave para desarrollar hábitos saludables que determinarán en gran medida la salud en la vida adulta.

Con respecto a las acciones del profesional de enfermería en el entorno escolar, tiene su papel fundamental en potenciar conductas protectoras para la salud. Así lo evidencia Costa et al. ${ }^{(13)}$ en una revisión bibliográfica en Brasil que muestra una relación entre la Estrategia de Salud Familiar y la escuela, con un enfoque en la labor del profesional de enfermería desde el punto de vista de prevención, promoción y atención de salud de niños, adolescentes y jóvenes; la cual tiene un papel clave en la difusión del conocimiento sobre la salud a través de estrategias lúdicas y creativas, que permite una mayor interacción y transmisión de conocimiento, además del fortalecimiento de relaciones, para prevenir la aparición de problemáticas de salud.

Entre los países donde se han implementado políticas sobre el rol de la enfermería escolar, basados en un estudio realizado por Kivimaki et al. ${ }^{(14)}$, destaca Finlandia, donde a través de una encuesta a 534 adolescentes sobre la facilidad de acceder a una enfermera escolar, la mayoría respondió que fue fácil su accesibilidad, ya que en este país es una recomendación nacional tener a una enfermera a tiempo completo o parcial en el establecimiento educacional.

Adicionalmente en el ámbito de salud, la promoción acoge los lineamientos de la OMS, que en cada país recoge posturas políticas con intencionalidades propias, pero tienen similitudes: las escuelas promotoras de salud son un compromiso para formar alianzas y optimizar recursos con una acción integrada de escuelas, comunidades y servicios de la salud. Además, son una herramienta política de promoción de la salud en el ámbito escolar. Por último, la escuela debe ser vista como un espacio productor de la salud, garantizando ambientes saludables y el desarrollo del buen vivir. Es así como en un estudio realizado por Hernández et al. ${ }^{(10)}$, determinaron las tendencias teóricas de la promoción de la salud en el ámbito escolar, tales como la visión de la escuela como lugar propicio para el cuidado de la salud de los escolares, la educación en salud para el autocuidado y fomento de estilos de vida y las escuelas promotoras de salud como un trabajo intersectorial para el desarrollo de políticas, conocimientos y ambientes. Así mismo en Latinoamérica, gradualmente se fueron incorporando a los postulados internacionales, las iniciativas locales de países, quienes desarrollaron una serie de normativas y documentos oficiales para la implementación de la promoción de la salud en el ámbito escolar. Tal como por ejemplo Colombia, Brasil y Cuba, desarrollaron una serie de normativas y documentos oficiales para la implementación de la promoción de la salud en el ámbito escolar. Se destaca la promoción de la salud en el ámbito escolar como una 
visión integral y multidisciplinar que considera el contexto familiar, comunitario, social y medioambiental. En este sentido, Lusquinos et al. ${ }^{(4)}$, en un estudio realizado en Portugal, evidencia que las directrices ministeriales son acordes a los lineamientos internacionales y realizan un trabajo en conjunto para desarrollar competencias en salud para los niños y jóvenes.

Particularmente en Chile un estudio realizado por Ceballo et al. ${ }^{(15)}$, en Concepción en el año 2020 demostró que no existen políticas públicas establecidas sobre las enfermeras escolares, y muestra la necesidad de la enfermera escolar, manifestando que una de las principales preocupaciones del Ministerio de Salud es el aumento de las enfermedades de transmisión sexual, donde la enfermera escolar está directamente relacionada en la educación al alumnado con énfasis preventivo.

Esta necesidad se puede demostrar con una investigación realizada por Gaete et al. ${ }^{(14)}$ en Valparaíso, Chile los cuales evaluaron la influencia de las conductas promotoras de salud de los padres en la de sus hijos adolescentes, la cual dio como resultado que las conductas promotoras de salud están directamente relacionadas entre las conductas presente en los padres y los adolescentes, ya que las costumbres de sus principales vínculos serán adoptadas en el periodo de la adultez, por lo tanto, es importante la presencia de un profesional competente que eduque a la comunidad escolar y oriente en conductas promotoras de salud.
2. Competencias y acciones del profesional de Enfermería en los establecimientos educacionales

Cabe mencionar que el análisis de las categorías se subdivide en 3 subcategorías: competencias cognitivas, habilidades técnicas y acciones/actividades de los profesionales de enfermería en los establecimientos educacionales las cuales se presentan a continuación.

Los profesionales de enfermería se desempeñan en seis áreas, según Torres et al. ${ }^{(16)}$, estas son: asistencial, educación, investigación, gestión, sociopolítico y emancipatorio. Estas áreas se desarrollan según la regulación de cada país sobre la actividad de los profesionales de enfermería en el ámbito escolar.

En primer lugar, dentro de unos de los quehaceres fundamentales de enfermeras escolares se encuentra la educación. Los programas educativos se consideran como positivos en las intervenciones de profesionales de enfermería. Es así como un estudio realizado por Soto et al. ${ }^{(17)}$, con el objetivo de conocer la educación en salud como parte del rol de enfermería en su quehacer profesional, destacó el rol educativo en diferentes niveles de atención y prevención en salud, donde la enfermera educa no solo al paciente, sino que involucra a la familia y comunidad. Las intervenciones están enfocadas en la promoción, prevención, protección, recuperación y rehabilitación de la salud. Por lo tanto, dentro de la formación debe haber un enfoque en el cual potenciar los conocimientos y habilidades de los futuros profesionales de enfermería. 
Beneficios del rol de enfermería en salud escolar...

Prosiguiendo con el quehacer educacional, uno de los aspectos más relevantes que se encuentran en los artículos es la educación temprana de las enfermedades de transmisión sexual en los escolares, teniendo su énfasis sobre todo en la etapa de la adolescencia. En un estudio realizado por Beech et al. ${ }^{(18)}$, en Londres, con el objetivo de explorar el papel y las actividades del servicio de enfermería escolar en salud sexual, las derivaciones de embarazos de adolescentes sugieren un apoyo por parte del servicio de enfermería escolar. De las derivaciones recibidas, solo el $15 \%$ fueron de sexo masculino y el $85 \%$ de estas se relacionaban con un problema en el uso de preservativo como método anticonceptivo. En cuanto a la edad, el $70 \%$ eran jóvenes entre los 13 y 15 años y los niños menores de 12 años representaron solo el 10\%. Esto resalta la necesidad de que los niños en edad escolar primaria incorporen información apropiada para su edad sobre la educación sexual integral y las relaciones saludables. Los servicios de enfermería escolar tienen un papel en la protección y derivación de los estudiantes a otros servicios, además de promover el bienestar infantil y su protección contra daños.

Desde otro punto de vista, en el ámbito nutricional, en un estudio realizado por Berradre et al. ${ }^{(19)}$, en Madrid España, con el objetivo de establecer el grado de conocimiento y cumplimiento del documento de consenso del Sistema Nacional de Salud sobre la alimentación en los centros educativos, a través de un estudio transversal con una muestra aleatoria de 182 establecimientos educacionales, se calculó que el $65,5 \%$ desconocían el documento. Junto con ello, el 70,3\% tenía identificada una persona que supervise el menú escolar. De estas personas, el $54,8 \%$ de los responsables tenían formación acreditada en dietética y nutrición. Por lo tanto, no solo los profesionales de enfermería tienen un enfoque en la promoción y prevención de la salud, sino que otros profesionales como los nutricionistas también pueden ingresar en el área de alimentación de los establecimientos educacionales.

Competencias cognitivas: Las competencias cognitivas que deben poseer los profesionales de enfermería escolar se describen a continuación. Un estudio español realizado por Alba et al. ${ }^{(11)}$, identifica que los conocimientos deben basarse principalmente en la atención primaria y comunitaria, urgencias, curso de adaptación pedagógica, pediatría y salud mental. Otro estudio realizado por Mori et al. ${ }^{(20)}$, en Perú, afirma que dentro de las competencias de la enfermera escolar está la educación para la salud, en donde se conjuga el aprender académico escolar y el aprender para la vida, salud y bienestar. En el contexto de los programas de salud escolar son ejecutados por profesionales de la salud con un enfoque en la promoción de la salud, estilos de vida saludable, vacunación, crecimiento y desarrollo, atención de adolescentes y detección temprana de los problemas más frecuentes.

Una habilidad cognitiva que deben desarrollar es la de generar investigación y puesta en marcha de nuevas intervenciones basadas en la evidencia y según el contexto inmerso, es así como un estudio brasileño, realizado por Da Silva et al. ${ }^{(21)}$, realizó una dinámica anti-bullying, que 
involucra las áreas psicosociales de los estudiantes, presentando diferencias significativas de disminución en la agresión indirecta y la victimización directa en el grupo que recibe la intervención versus el grupo control.

Otra habilidad cognitiva a desarrollar es la interacción con el individuo, su entorno y la realización de intervenciones lúdicas, un estudio de revisión bibliográfica de las regiones del caribe y américa latina, realizado por Costa et al. ${ }^{(13)}$, evidencia que es notoria la importancia de acciones lúdicas que faciliten el aprendizaje de los alumnos y la importancia de una alimentación sana, buscando una mejora en el estado nutricional, permitiendo una mayor interacción y fortalecimiento de las relaciones, satisfaciendo de manera oportuna sus necesidades.

Por otro lado, otra de las competencias de la enfermería escolar en el quehacer de la investigación a desarrollar es sobre la temática del desarrollo psicomotor (DSM) y las dificultades del aprendizaje en preescolares con probable trastorno por déficit de atención e hiperactividad (TDAH). Un estudio epidemiológico realizado por Marín et al. ${ }^{(22)}$, en España, demostró que un $34 \%$ de los niños con probable TDAH muestran dificultades en el aprendizaje referido por sus padres. En el estudio analítico se asoció que las dificultades de aprendizaje se dan por la presencia de un retraso en el DSM en los 3 primeros años de vida. Por lo tanto, según los resultados, es importante la detección precoz del TDAH en los preescolares para iniciar de forma temprana intervenciones por profesionales capacitados y la educación debe ser desarrollada de la manera más efectiva.

Por último, desde la perspectiva de las propias enfermeras escolares en un estudio realizado por Kobosab et al. ${ }^{(23)}$, en EE.UU., se evaluaron 230 enfermeras escolares de 17 centros, para establecer el conocimiento real y percibido de la diabetes mellitus tipo 1. La mayoría de las enfermeras escolares percibieron mayor su conocimiento real que el demostrado, evidenciando que las competencias cognitivas deben estar en constante desarrollo y actualización.

Habilidades técnicas: En relación a las habilidades técnicas de los profesionales de enfermería, Hoekstra et al. ${ }^{(24)}$, en Inglaterra, señalan en el área asistencial la ejecución de las inmunizaciones, detección de problemas de salud y necesidades de salud sexual en adolescente, avalado por un estudio peruano, realizado por Mori et al. ${ }^{(20)}$.

Continuando con esta mirada en un artículo de revisión narrativa de Latinoamérica, realizado por Hernández et al. ${ }^{(10)}$, se evidencia que lo primordial son las intervenciones enfocadas en los estilos de vida con dirección biologicista e higienista. En cuanto a las acciones educativas, en su mayoría se orientaron a la identificación de factores de riesgo, la socialización de procesos fisiológicos o patológicos, y en muy pocos casos, se encontraron acciones orientadas al desarrollo de habilidades psicosociales para la vida, autoestima y relaciones interpersonales. Otra revisión sistemática brasileña realizada por Costa et al. ${ }^{(13)}$, evidencia que las necesidades a cubrir en 
Beneficios del rol de enfermería en salud escolar...

los estudiantes se relacionan con la higiene, pediculosis, caries, retraso en vacunas y malnutrición.

Acciones y actividades: Entre las acciones $\mathrm{y}$ actividades a las que se dedican los profesionales de enfermería escolar, describen lo siguiente: Hoekstra et al. ${ }^{(24)}$, señalan que deben presentar un manejo de la protección de la infancia, administrar clínicas, realizar sesiones sin cita previa, hacer y responder a referencias, brindar sesiones de inmunización, apoyar a los estudiantes con necesidades de salud sexual, realizar sesiones de detección de salud, incluido el Programa Nacional de Medición Infantil, apoyando a los escolares con problemas de salud y trabajando con las familias para satisfacer las necesidades, es decir, redactar planes de atención, promoción y prevención en salud. En relación a las acciones de promoción y prevención de salud, en México, García et al. ${ }^{(12)}$, concluyen que las enfermeras escolares intervinieron en la promoción de hábitos saludables para escolares y padres, evidenciando que $63,04 \%$ reportó haber generado un cambio en sus hábitos después de asistir a los talleres, lo cual repercute directamente en la salud de los escolares y de sus familias de forma positiva. De esta misma forma en un estudio peruano, realizado por Mori et al. ${ }^{(20)}$ destaca una acción intersectorial salud-educación y políticas de promoción de salud de los escolares dentro las instituciones educativas.

El enfoque educativo de las enfermeras escolares a través de la promoción de hábitos saludables estableció su rol, en un estudio realizado por Martil et al. ${ }^{(25)}$, en España, en el cual se efectuó una intervención educativa de tres talleres con el objetivo de promover hábitos saludables: hábitos alimentarios, actividad física y sueño-descanso a través de un profesional de enfermería escolar. Estas temáticas fueron pesquisadas mediante una entrevista la cual evidenció que el 16,9\% de los escolares presentó un patrón de alimentación ineficaz del niño y el $26,2 \%$ presentó un estilo de vida sedentario, este último post intervención presentó un $50 \%$ menos de casos. El $17,8 \%$ de los escolares tenía un deterioro del patrón del sueño de los cuales post intervención disminuyeron $59 \%$ de los casos, evidenciando un impacto significativo en el cambio de estilos de vida sedentario y la mejora del patrón del sueño posterior a la intervención educativa de las enfermeras.

Otra de las acciones desempeñadas por el profesional de enfermería es la aplicación y capacitación de reanimación cardiopulmonar (RCP), según lo descrito por Beech et al. ${ }^{(18)}$. La participación de la enfermera escolar en estos requisitos de capacitación en reanimación cardiopulmonar (RCP) recientemente obligatorios es una oportunidad para que sean parte del éxito académico de los estudiantes para mejorar la salud de las comunidades. Se recomienda que la enfermera de la escuela enseñe a los padres reanimación cardiopulmonar (RCP) con las manos al comienzo del año escolar. Se concluye que deben aumentar su visibilidad mostrando el impacto que pueden tener en nuestras escuelas y comunidades. Las capacitaciones de las enfermeras escolares en los docentes permiten aumentar los niveles de conocimiento y enfrentar situaciones de 
riesgo en los escolares. Otro estudio que sustenta las capacitaciones que realizan las enfermeras escolares, realizado por Martín et al. ${ }^{(26)}$, en España, a los docentes, permite aumentar el nivel de conocimiento de primeros auxilios, ya que previamente tenían un déficit de conocimiento de ello, por ejemplo, curar heridas con alcohol.

Dentro del accionar de enfermeras escolares, se identifican ciertas características que mejoran la efectividad de las intervenciones educativas en los establecimientos educacionales. Esto se refleja en los resultados de Kogolu et al. ${ }^{(27)}$, en Turquía, donde con un total de 477.163 participantes escolares pertenecientes a 933 escuelas recibieron servicios de salud escolar en los años 2009-2010 proporcionados por enfermeras a tiempo completo. Se evaluaron los beneficios entre instituciones con o sin presencia de la enfermera escolar. La principal intervención de enfermeras escolares fue la vigilancia con un $41,9 \%$ seguido de la educación con un $40,1 \%$, tratamientos y procedimientos corresponden a un $9,7 \%$ y la gestión a un $8,3 \%$. Como resultado de estas intervenciones, se produjo un aumento significativo en la calidad de vida de los estudiantes. Cabe destacar que no se encontró una relación entre el número de ausentismo y las intervenciones realizadas por enfermeras escolares, por el contrario, si hubo un aumento significativo del rendimiento escolar y una disminución de la procrastinación.

Para finalizar, las competencias y actividades de las enfermeras escolares se basan en la promoción de la salud y prevención de enfermedades de la salud. Además, tienen su enfoque sobre todo en el área de educación e investigación para satisfacer las necesidades que presenta la comunidad escolar, desempeñando un rol fundamental, sustentado en bases de conocimientos científicos y humanistas para brindar una atención integral con el objetivo de contribuir a mejorar el bienestar de las personas en la sociedad.

\section{Percepciones de la comunidad escolar frente a la enfermería profesional en salud escolar}

El rol de la enfermera escolar puede ser visualizado por diferentes participantes de la comunidad escolar. Es por ello que es importante conocer ambas perspectivas: de los que entregan la atención de salud y de los que la reciben. En un estudio en Trujillo, Perú (Mori et al. $\left.{ }^{(20)}\right)$ los directores de las instituciones educativas coinciden en que tener una enfermera en la escuela brinda seguridad y mejora los hábitos a los alumnos, profesores y a los padres de familia. En la misma línea, pero desde la percepción de los funcionarios de una escuela, Ceballo et al. ${ }^{(15)}$, en Concepción (Chile), señalan que la relevancia de la enfermera escolar brinda ayuda en las problemáticas más frecuentes: caídas y vómitos. La acción percibida más relevante fue la atención de primeros auxilios y la de menor importancia fue la acción de realizar estudios científicos para conocer actitudes y prácticas inadecuadas en los estudiantes. Se concluye que el rol asistencial es el más necesario que debe desarrollar la enfermera escolar.

En relación a lo anterior, y desde la perspectiva de los docentes, Gómez et al. ${ }^{(6)}$, en España, señalan que la mayoría de los docentes se sienten inseguros y temerosos en el abordaje de los escolares 
Beneficios del rol de enfermería en salud escolar...

con patologías crónicas, por miedo a no saber qué hacer y/o causar más daño en alguna situación de gravedad. Docentes reclaman la presencia de una enfermera escolar para atender a los niños con enfermedades crónicas, argumentando no tener las capacidades ni obligación de asumir dichas responsabilidades. Siguiendo con la perspectiva de los docentes, estudios desarrollados por Bayik et al. ${ }^{(5)}$, en Turquía, sostienen que la mayoría (94,5\%) informó la importancia de que una enfermera escolar sea empleada en un establecimiento educacional como parte del programa de salud integral. Sumado a esto, casi todos los padres $(96,5 \%)$ afirmaron que debería emplearse una enfermera escolar en todas las escuelas.

Davó et al. ${ }^{(28)}$, en España, se evaluaron grupos de discusión del profesorado de 21 escuelas primarias, los cuales manifestaron que se les delega mayores funciones de las que deberían tener. Del mismo modo, perciben que se intenta delegar los temas de salud en asignaturas de menor peso curricular. El profesorado muestra desinterés en las campañas de salud, ya que no responden adecuadamente a las necesidades reales del alumnado; a diferencia de las actividades que cuentan con una planificación previa para cada año escolar, las cuales cumplen adecuadamente con los temas de salud. Bajo esta dinámica, reconocen que su bajo conocimiento en salud dificulta alcanzar las metas de la educación en salud.

Desde la opinión de equipos directivos, Álvarez et al. ${ }^{(29)}$, en España, señalan que existe una tranquilidad y calidad en salud al contar con profesionales de enfermería en los establecimientos educacionales. Sin embargo, en algunos casos perciben que su rol va más allá de un beneficio y se plantea como una necesidad en los establecimientos que reciben estudiantes con necesidades especiales. Con respecto a la opinión de familias, manifiestan que la presencia de profesionales de enfermería escolar es una tranquilidad en cuanto a salud, al proporcionar seguridad y dar respuesta profesional a temas de salud, además es un plus para los establecimientos educacionales, ya que incluso es promocionado a través de páginas web de los establecimientos y es el tema que causa mayor curiosidad ante los padres. Reconocen beneficios tanto a nivel sanitario como social y agradecen la gestión de pequeñas urgencias. Junto con esto, se sigue la misma perspectiva de las familias, en resultados obtenidos por Montoro et al. ${ }^{(30)}$, en España, quienes compararon centros educativos sin enfermera escolar y con enfermera escolar. La mayoría de los padres tomaron en cuenta la presencia de la enfermera escolar en el establecimiento y reconocen en ella la importancia de la atención y educación en salud.

\section{Demanda de prestaciones en salud de los profesionales de Enfermería en salud escolar}

Según la demanda de las prestaciones de los profesionales de Enfermería en salud escolar se ha dividido en dos subcategorías: Tipo de consulta y consulta según área geográfica.

Tipo de consultas más demandadas para la enfermera en salud escolar en los establecimientos escolares. Las principales demandas de atención de enfer- 
mería en salud escolar, fueron reveladas en un estudio realizado en Brasil por Oliveira et al. $^{(31)}$, en donde se señala que la demanda fue mayor entre quienes sufrieron alguna lesión $(60,8 \%)$, violencia física $(63,7 \%)$, asma o sibilancias $(66,2 \%)$, dolor de muelas $(59,5 \%)$ y quienes se sentían solos $(59,8 \%)$. Por otro lado, la demanda fue menor entre quienes presentaban malnutrición por déficit o exceso $(51,2 \%)$ y entre aquellos que calificaron su estado de salud como muy bueno $(54,1 \%)$. En este mismo sentido, un estudio realizado en Sao Paulo, Brasil, según la Encuesta Nacional de Salud del año 2015, evidencia que la mayor demanda de atención fue por parte de adolescentes entre 13 a 17 años (56,7\%), principalmente mujeres $(61,1 \%)$, y estudiantes de escuelas privadas $(69,9 \%)$.

\section{Tipo de demanda según zona geográfica.}

La demanda de una enfermera, según la zona geográfica, varía, lo cual se observó en un estudio realizado en dos escuelas canadienses, por Sanders et al. ${ }^{(32)}$, en donde se comparó la intervención de una enfermera en el área urbana y en el área rural. En esta última, previo a la realización de la intervención fue necesario invertir tiempo para desarrollar relaciones con los padres y la comunidad escolar, para así formar un comité de salud que pueda usar la herramienta de evaluación y comenzar a abordar la salud en la escuela. Por otra parte, en la escuela ubicada en la zona urbana, ya existía un comité activo de salud dentro de la escuela, por lo cual la participación estudiantil fue activa y el proceso de intervención fue logrado en menor tiempo, revelando así las diferencias entre cada contexto geográfico.

\section{CONCLUSIONES}

El entorno escolar no debe limitarse exclusivamente a un espacio de procesamiento de conocimiento intelectual, es correcto decir que este conocímiento es fundamental para construir las habilidades y capacidades de los individuos, sin embargo, es insuficiente cuando no está acompañada de la promoción integral de la salud. La integralidad en el cuidado de la salud debe ser desarrollada en asociación con el profesional de enfermería, quien debe realizar intervenciones que fomenten el bienestar físico, emocional y psicosocial en la comunidad escolar.

No existe regulación de la enfermería escolar en todas las comunidades por igual. En algunos países europeos existen políticas públicas establecidas sobre el rol de la enfermería escolar en salud y hay una recomendación nacional de tener una enfermera escolar a tiempo completo o parcial. En Latinoamérica en muy pocos casos se ha propiciado la incorporación de acciones por profesionales de salud a los proyectos educativos.

En Chile, no existe una regularización de la figura de la enfermera escolar ya que está ligada a la salud comunitaria y familiar que depende exclusivamente de la atención primaria de salud, por lo tanto, se plantea como una necesidad. Ante esto, se propone un plan de regularización total para los establecimientos educacionales a través de una nueva política pública en un proyecto 
Beneficios del rol de enfermería en salud escolar...

de ley que permita incorporar una enfermera en cada escuela pública y privada para fortalecer el primer nivel de atención y la ampliación de los espacios de práctica profesional de la enfermera. Para fortalecer el área de investigación se proponen más publicaciones sobre enfermería en el ámbito escolar en Latinoamérica. Se destacan evidencias científicas sobre el tema de promoción de salud escolar publicadas en Brasil, entre las que más publicaciones presentan sobre este tema.

Las principales competencias recopiladas muestran que las intervenciones del profesional de enfermería están orientadas a la promoción de estilos de vida saludable, prevención, protección de la infancia, recuperación y rehabilitación de la salud de la comunidad escolar, realizar sesiones sin cita previa, control de crecimiento y desarrollo, atención de adolescentes, educación sexual y detección de salud de las principales problemáticas, inmunización, urgencias, curso de adaptación pedagógica considerando a los escolares desde una mirada holística. La integración de un profesional a la comunidad escolar permite enfrentar problemáticas mediante acciones de promoción y prevención de la salud realizando controles de salud escolar, vacunación, educación sobre nutrición, sexualidad e higiene, como también prevención y atención de accidentes dentro del establecimiento. Esta visión no solo es de los funcionarios pues, a opinión de ellos, los padres y sus familias también la consideran un recurso vital que debe estar presente en los establecimientos educacionales, cuyo valor está en el trabajo integrado que puede realizar en la comunidad escolar, en coordinación con los profesionales de los centros de salud primarios.

Por último, enmarcado en la pandemia covid-19 presentada desde el año 2019 al presente 2021, se presenta una situación particular del retorno escolar de los estudiantes a los establecimientos educacionales. Los escolares deberán volver a sus clases presenciales, según la evolución de la pandemia, y deberán adoptar medidas de prevención para desarrollar su educación a través de los años. Pero, esto no solo involucra a los estudiantes, sino a toda la comunidad escolar: personal del colegio, apoderados y equipo gestor. Frente a esta situación el profesional de enfermería podría desarrollar una función relevante en promoción y prevención, participar activamente en el proceso de vacunación, gestión y aplicación de las medidas de retorno en contexto de pandemia para toda la comunidad educativa. Situación que demanda un personal de salud capacitado, como el profesional de enfermería escolar, que pueda orientar de la mejor forma los protocolos de retorno escolar en una pandemia como el covid-19, y sobre todo pueda educar a la comunidad escolar, ya que el profesional de enfermería cuenta con una formación que le permite brindar cuidados a todas las personas que pertenecen a la comunidad educativa.

\section{REFERENCIAS BIBLIOGRÁFICAS}

1. Omar Martínez V. La enfermera escolar: situación actual y necesidades de la población infantil. Ocronos [Internet]. 2020. [consultado 7 junio 2020]; (2603-8358):1-5. Disponible en: 
Cáceres Pérez MF, Castillo Castillo NI, Salinas Vergara CF, Bianchetti Saavedra AF, Vega

Flores RI

https://revistamedica.com/enfermeraescolar-necesidades-poblacion-infantil/

2. Ministerio de Salud. 2021. Promoción de la salud [Internet] [consultado el 11 marzo 2021] Disponible en: https://www.minsal.cl/wpcontent/uploads/2018/09/ANEXO-10PROMOCION-DE-LA-SALUD.pdf

3. Minsal. 2021. Promoción de salud [Internet]. [consultado el 11 marzo 2021]

Disponible

en: https://www.minsal.cl/wpcontent/uploads/2016/09/1_PROMOCI ON-DE-SALUD.pdf

4. Lusquinhos L., Carvalho G. Educación sanitaria en las escuelas portuguesas: directrices para los sectores de la salud y la educación. Revista de enfermería [Internet] 2019. [consultado 25 agosto 2020];4(21):2. Disponible en:

https://rr.esenfc.pt/rr/index.php?modul $\mathrm{e}=\mathrm{rr} \&$ target $=$ publicationDetails \&pesq uisa $=\& i d \_$artigo $=3130 \& i d \_r e v i s t a=24$ \&id_edicao=179 DOI: 10.12707/RIV19020

5. Bayik A, Gulizar J, Kalkim A, Muslu L, Yildirim N. Parents' and teachers' expectations of school nurse roles: A scale development study. International Journal of Nursing Sciences [Internet]. 2017. [consultado 22 junio 2020];(3):1-2. Disponible en:

https://pubmed.ncbi.nlm.nih.gov/3140 $6757 /$

DOI: 10.1016/j.ijnss.2017.05.002

6. Gómez G., Carollo M., Gómez A., Sánchez L., García F., Rodríguez A. Escolares con enfermedades crónicas, ¿qué les preocupa a sus profesores? Anales de pediatría [Internet] 2020. [consultado el 20 agosto 2020]; 93(6): 374-379. Disponible en:

https://www.sciencedirect.com/science /article/pii/S1695403320300795?via\% 3Dihub

DOI:10.1016/j.anpedi.2020.02.004

7. Del Val E. La enfermera en la escuela y la salud escolar [tesis fin de grado]. España, Cantabria. Universidad de Cantabria; 2015. 4-13p.

8. Díaz Portillo J. Guía práctica de lectura crítica de artículo científicos originales en ciencias de la salud. 1st ed. Madrid: Instituto Nacional de Gestión Sanitaria; 2012.

9. Manterola C, Zavando D. Cómo interpretar los "Niveles de Evidencia" en los diferentes escenarios clínicos. Revista Chilena de Cirugía. [Internet] 2009 [consultado el 20 agosto 2020];61(6): 582-595. Disponible en:

https://www.scielo.cl/scielo.php?s cript=sci_arttext\&pid=S071840262009000600017 DOI: 10.4067/S0718-40262009000600017

10. Hernández-Sánchez J, Oviedo-Cáceres M, Rincón Méndez A, Hakspiel-Plata M, Mantilla Uribe B. Tendencias teóricas y prácticas de la promoción de la salud en el ámbito escolar en Latinoamérica. Universidad Industrial de Santander [Internet] 2019 [consultado 22 Agosto 2020]; 51(2):155-168. Disponible en: http://www.scielo.org.co/scielo.php?sc ript=sci_arttext\&pid=S0121$08072019000200155 \& \operatorname{lng}=\mathrm{en} \& \mathrm{nrm}=$ is o\&tlng=es

11. Fernández C, Casal L, Cebreiro B, Tizón E, Martínez E. La Enfermería escolar: contenidos y percepciones sobre su pertinencia en las escuelas 
Beneficios del rol de enfermería en salud escolar...

inclusivas. Enfermería global [Internet]. 2019 [consultado 20 junio 2020]; 18(4):291-307. Disponible en: https://revistas.um.es/eglobal/article/vi ew/344611

DOI: 10.6018/eglobal.18.4.344611

12. Garcia X.,Allué N., Pérez A., Ariza C., Sánchez F., López M., Nebot M. Hábitos alimentarios, conductas sedentarias y sobrepeso y obesidad en adolescentes de Barcelona. Anales de Pediatría [Internet]2015[consultado el 25 agosto 2020];83(1):3-10. Disponible en:

https://www.sciencedirect.com/science /article/pii/S1695403314003877?via\% 3Dihub DOI: 10.1016/j.anpedi.2014.07.006

13. Costa E, Oliveira E, Silveira A, Mombaque W. Educación para la salud en el contexto escolar: estudio de revisión integradora. Enfermería global [Internet].2017[consultado 22 junio 2020];1695-6141(46):3-11. Disponible en:

https://scielo.isciii.es/pdf/eg/v16n46/1 695-6141-eg-16-46-00464.pdf DOI: 10.6018/eglobal.16.2.235801

14. Gaete J., Rojas C., Olivares E., Chen M. Influencia de las conductas promotoras de salud de los padres en la de sus hijos adolescentes. Rev. méd. Chile [Internet]. 2016 [consultado 14 junio 2020]; 144(7): 870-878. Disponible en: https://www.scielo.cl/scielo.php?script =sci_arttext\&pid=S0034-

98872016000700007 DOI: 10.4067/S0034-98872016000700007

15. Ceballo B., Fonseca D., Fuenzalida N, Morales L., Parada D., Morales I. Importancia de la enfermera escolar según la percepción de funcionarios de colegios básicos de una provincia de Chile. Ciencia y Enfermería [Internet] 2020 [consultado el 24 de agosto 2020];26:5. Disponible en: https://www.scielo.cl/scielo.php?script $=$ sci_arttext\&pid=S0717-

95532020000100204\&lang=pt DOI: 10.4067/s0717-95532020000100204

16. Torres E. Patrón de conocimiento emancipatorio y su contribución al desarrollo del conocimiento de enfermería. Cultura del cuidado [Internet] 2017 [consultado el 24 de agosto 2020] 14(1): 2-5. Dsiponible en: https://revistas.unilibre.edu.co/index.p $\mathrm{hp} /$ cultura/article/view/4330 DOI: 10.18041/17945232/cultrua.2017v14n1.4330

17. Soto M., Masalan P., Barrios S. La educación en salud, un elemento central del cuidado de enfermería. Rev Médica Clínica Las Condes. [Internet] 2018 [consultado el 20 agosto 2020]; 29(3):288-300. Disponible en:

https://www.sciencedirect.com/science /article/pii/S0716864018300543?via\% 3Dihub

DOI: 10.1016/j.rmclc.2018.05.001

18. Beech S., Sayer L. Analysis of sexual healthcare provided by school nurses [Internet]. 2017 [consultado el 23 de agosto 2020]; 19 (3): 288-300. Disponible en: https://pubmed.ncbi.nlm.nih.gov/2924 8022/ DOI: 10.1017/S1463423617000780

19. Berradre B., Royo M., Bosqued M., Moya M., López L., Menú escolar de los centros de enseñanza secundaria de Madrid: conocimiento y cumplimiento de las recomendaciones del Sistema Nacional de Salud. Gaceta Sanitaria 
Cáceres Pérez MF, Castillo Castillo NI, Salinas Vergara CF, Bianchetti Saavedra AF, Vega

Flores RI

[Internet] 2015 [consultado el 20 de agosto 2020]; 29 (5): 341-346. Disponible en: https://www.sciencedirect.com/science /article/pii/S021391111500076X?via\% 3Dihub DOI: 10.1016/j.gaceta.2015.04.009

20. Mori F., Bustamante S., Leitón Z, Santillán R. Competencias de la enfermera en instituciones educativas: una mirada desde los gestores educativos. Revista Gaúcha de Enfermagem [Internet]. 2017 [consultado 21 junio 2020];152(1):3-7. Disponible en: https://www.scielo.br/j/rgenf/a/kFzdcB dx8DdfgQ7pwZXB4yP/?format=pdf\& lang=es $\quad$ DOI: $\quad 10.1590 / 1983-$ 1447.2018.2017-0152

21. Da Silva L., Da Silva J., Vilela A., Silva F., Malta F., Tossi M, Theater of the Oppressed and bullying: nursing performance in school adolescent health. Revista brasileira de Enfermagem[Internet];2020[consultad

o el 20 agosto 2020];73(1):2-5. Disponible en : https://www.scielo.br/j/reben/a/gV5QT pkxzmFmCHch3Hvc4LD/?format=pdf \&lang=en DOI: $10.1590 / 0034-7167-$ 2017-0910

22. Marín M., Borra M., Álvarez M., Soutullo C. [Internet] Desarrollo psicomotor y dificultades del aprendizaje en preescolares con probable trastorno por déficit de atención e hiperactividad. Estudio epidemiológico en Navarra y La Rioja [Internet] 2017 [consultado el 22 agosto 2020]; 32(8):487-493. Disponible en: https://www.sciencedirect.com/science /article/pii/S0213485316001183?via\%

3Dihub

DOI: 10.1016/j.nrl.2016.02.009

23. Kobosab E., Imiela J., Kryczka T., Szewczyk A., Knoff B. Actual and perceived knowledge of type 1 diabetes mellitus among school nurses. Nurse Education[Internet]. 2020 [consultado el 20 de agosto 2020]; 87(104304):6-8. Disponible en: https://www.sciencedirect.com/science /article/pii/S0260691719305118?via\% 3Dihub

DOI: 10.1016/j.nedt.2019.104304

24. Hoekstra B., Young V., Eley C., Hawking M., McNulty C., School Nurses' perspectives on the role of the school nurse in health education and health promotion in England: a qualitative study. BMC Nurs [Internet]. 2016 [consultado el 20 agosto 2020]; 15 (73):1-5. Disponible en: https://pubmed.ncbi.nlm.nih.gov/2805 0164/ DOI:10.1186/s12912-016-0194$\mathrm{y}$

25. Martil Marcos DM, Calderón García S, Carmona Sánchez A, Brito Brito PR. Efectividad de una intervención enfermera comunitaria en el ámbito escolar para la mejora de hábitos alimentarios, de actividad física y de sueño-descanso. ENE revista de enfermería [Internet]. 2019 [consultado 20 junio 2020];13(2):13-18. Disponible en: https://scielo.isciii.es/scielo.php?script =sci_arttext\&pid=S1988348X2019000200002\&lang=es\#B1

26. Martín A. Educación para la salud en primeros auxilios dirigida al personal docente del ámbito escolar. Enfermería universitaria [Internet] 2015 [consultado el 22 agosto 
Beneficios del rol de enfermería en salud escolar...

2020];12(2):88-92. Disponible en: http://www.scielo.org.mx/pdf/eu/v12n 2/1665-7063-eu-12-02-00088.pdf DOI: 10.1016/j.reu.2015.04.004

27. Kogolu D, Emiroglu ON. The Impact of Comprehensive School Nursing Services on Students' Academic Performance. Journal of Caring Sciences [Internet]. 2017 [consultado 21 junio 2020]; 6(1):9-14. Disponible en:

https://www.ncbi.nlm.nih.gov/pmc/arti cles/PMC5348663/ DOI: 10.15171/jcs.2017.002

28. Davó M., García M., La Parra D., Educación para la salud en la escuela primaria: opinión del profesorado de la ciudad de Alicante. Gaceta Sanitaria. [Internet] 2016 [consultado el 20 agosto 2020]; 30(1):31-36. Disponible en: https://www.sciencedirect.com/science /article/pii/S0213911115001508?via\% 3Dihub

DOI:10.1016/j.gaceta.2015.07.008

29. Álvarez R., Eguilaz M., Miguel S. La Enfermería Escolar: Un recurso necesario para la comunidad educativa. Revista de educación [Internet] 2018 [consultado el 15 Junio 2020];1(22):167-178. Disponible en: https://publicaciones.unirioja.es/ojs/ind ex.php/contextos/article/view/3374

DOI: $10.18172 /$ con.3374.

30. Montoro García A, Fernández Gutiérrez P. La enfermera escolar.
Opinión de los progenitores de niñas y niños que inician la escolarización. Metas de enfermería [Internet]. 2018 [consultado 25 junio 2020];20(10):8-9. Disponible en: https://www.enfermeria21.com/revista s/metas/articulo/81166/ DOI: 10.35667/MetasEnf.2019.20.10030811 66

31. Oliveira Max Moura de, Andrade Silvânia Suely Caribé de Araújo, Stopa Sheila Rizzato, Malta Deborah Carvalho. Demand for health services or professionals among Brazilian adolescents according to the National School Health Survey. Rev. bras. epidemiol. [Internet]. 2018 [consultado 25 agosto 2020];21(1):2-4. Disponible en:

https://www.scielo.br/j/rbepid/a/7Fpfg YSPBgvXzfVhdmDgF6B/?lang=pt DOI:10.1590/1980549720180003.supl.1

32. Sanders T., O'Mahony J., Duncan S., Mahara S., Pitman V., Ringstad K., Weatherman K. Opening the doors for school health-An exploration of public health nurses' capacities to engage in comprehensive school health programs. Public Health Nursing. [Internet].2019 [consultado el 24 agosto 2020];36(3):348-356. Disponible en:

https://pubmed.ncbi.nlm.nih.gov/3088 3923/ DOI:10.1111/phn.12607 